\title{
Increasing objectively measured sedentary time increases clustered cardiometabolic risk: a 6 year analysis of the ProActive study
}

\author{
Katrien Wijndaele • Gillian Orrow • Ulf Ekelund • \\ Stephen J. Sharp • Søren Brage • Simon J. Griffin • \\ Rebecca K. Simmons
}

Received: 27 June 2013 / Accepted: 14 October 2013 / Published online: 6 November 2013

(C) The Author(s) 2013. This article is published with open access at Springerlink.com

\begin{abstract}
Aims/hypothesis We aimed to quantify the associations between change in objectively measured sedentary and moderate-to-vigorous physical activity (MVPA) times and self-reported television viewing over 6 years and change in a clustered cardiometabolic risk score (CCMR), including and excluding waist circumference (CCMR without adiposity component, $\mathrm{CCMR}_{\text {no adip }}$ ), and its individual components, among the adult children of people with type 2 diabetes.

Methods In 171 adults (mean \pm SD age $42.52 \pm 6.30$ years; $46 \%$ men) with a parental history of diabetes (ProActive UK), physical activity accelerometer measures and self-reported television viewing were assessed at baseline and a mean \pm $\mathrm{SD}$ of $6.27 \pm 0.46$ years later. Associations between change in sedentary time, MVPA time and television viewing and cardiometabolic risk and mediation by adiposity change were
\end{abstract}

Electronic supplementary material The online version of this article (doi:10.1007/s00125-013-3102-y) contains peer-reviewed but unedited supplementary material, which is available to authorised users.

K. Wijndaele $(\bowtie) \cdot$ U. Ekelund $\cdot$ S. J. Sharp $\cdot$ S. Brage $\cdot$

S. J. Griffin • R. K. Simmons

MRC Epidemiology Unit, Institute of Metabolic Science, University

of Cambridge School of Clinical Medicine, Box 285, Cambridge

Biomedical Campus, Cambridge CB2 0QQ, UK

e-mail: katrien.wijndaele@mrc-epid.cam.ac.uk

G. Orrow $\cdot$ S. J. Griffin

Department of Public Health and Primary Care,

University of Cambridge, Cambridge, UK

U. Ekelund

Department of Sport Medicine, Norwegian School of Sport Sciences,

Oslo, Norway examined by multiple linear regression and the product of coefficients method, respectively.

Results Greater increases in sedentary time (h/day) were associated with larger increases in clustered cardiometabolic risk (CCMR: 0.08 [95\% CI 0.01, 0.15]; CCMR ${ }_{\text {no adip }}$ : 0.08 [0.01, $0.16])$ and triacylglycerol $(0.15[0.01,0.29])$, independent of baseline sedentary and MVPA times, change in MVPA time and other confounders. No evidence was found for mediation by change in waist circumference and BMI for the associations with $\mathrm{CCMR}_{\text {no adip }}$ and triacylglycerol. Greater increases in MVPA time (h/day) were associated with larger decreases in waist circumference $(-3.86[-7.58,-0.14])$, independently of baseline MVPA and sedentary times, change in sedentary time and other confounders. Television viewing was not independently associated with any of the cardiometabolic outcomes.

Conclusions/interpretation Increasing sedentary time is independently related to increasing clustered cardiometabolic risk and triacylglycerol in adults at high risk of developing diabetes. Strategies to prevent diabetes might target reducing sedentary time.

Trial registration ISRCTN61323766

Keywords Adiposity - Cardiovascular disease risk .

Longitudinal study · Moderate-to-vigorous physical activity • Sedentary behaviour $\cdot$ Television viewing

$\begin{array}{ll}\begin{array}{l}\text { Abbreviations } \\ \text { CCMR }\end{array} & \begin{array}{l}\text { Clustered cardiometabolic risk score } \\ \text { Clustered cardiometabolic risk score } \\ \text { without adiposity component }_{\text {no adip }}\end{array} \\ \text { MET } & \text { Metabolic equivalent } \\ \text { MVPA } & \text { Moderate-to-vigorous physical activity } \\ \text { SES } & \text { Socioeconomic status }\end{array}$

Abbreviations

CCMR Clustered cardiometabolic risk score without adiposity component

MVPA Moderate-to-vigorous physical activity

Socioeconomic status 


\section{Introduction}

Central obesity, dysglycaemia, dyslipidaemia and hypertension are closely related cardiometabolic abnormalities that cluster in a large proportion of the adult population worldwide. This cluster of factors substantially increases the risk of developing type 2 diabetes, as well as cardiovascular and other chronic diseases [1, 2]. Insufficient moderate-to-vigorous physical activity (MVPA) is a recognised contributor to elevated cardiometabolic risk levels [3] and has been targeted by lifestyle interventions in adults at high risk of type 2 diabetes. However, such interventions have had limited success, resulting in relatively modest changes in MVPA [4].

Excessive time spent sedentary is associated with an increased cardiometabolic risk, independent of time spent in MVPA, and has been suggested as a potential target for lifestyle interventions [5-9]. Sedentary behaviour represents any waking behaviour characterised by an energy expenditure of $\leq 1.5$ metabolic equivalents (METs) while in a sitting or reclining posture [10]. Adults, on average, spend more than $7 \mathrm{~h}$ of the waking day sedentary [11], an amount that is relatively independent of the time spent in MVPA [12]. However, there is currently insufficient evidence to establish a longitudinal association between sedentary time and cardiometabolic risk [13]. The few longitudinal studies examining this hypothesis [14-17] have mainly focused on self-reported television viewing as a marker of leisure-time sedentary behaviour $[15,16]$. Although television viewing is a potentially interesting intervention target, it is only one aspect of sedentary time and its association with cardiometabolic risk may differ from that of overall sedentary time [7, 18].

Because individuals at high risk of diabetes experience difficulties in increasing their MVPA [4], identifying additional behavioural targets should inform the development of future preventive interventions in these populations. Longitudinal investigations incorporating objectively quantified time spent sedentary and in MVPA, as well as television viewing time, can provide novel insights into whether, and to what extent, changing these behaviours will independently impact cardiometabolic risk. Furthermore, elucidating whether these associations are predominantly explained by a change in adiposity or by alternative physiological processes will provide insight into the mechanism underlying these associations [19]. Central obesity is regarded as an important aetiological component in the clustering of elevated cardiovascular risk factors and may therefore act as a mediator [20].

We aimed to estimate the independent associations between changes in objectively measured time spent sedentary, in MVPA and in self-reported television viewing over 6 years and changes in clustered and individual cardiometabolic risk factors in adults with a parental history of type 2 diabetes. We also examined whether these associations were mediated by a change in central or general adiposity.

\section{Methods}

We performed a cohort analysis of the ProActive trial, the design of which is described in detail elsewhere [21]. In short, ProActive evaluated the efficacy of a theoretical, evidenceand family-based intervention programme that aimed to increase physical activity levels in adults who were at high risk of type 2 diabetes by virtue of having a parent with the disease. Out of 465 eligible individuals aged $30-50$ years, 365 were randomly assigned to one of three interventions delivered over 1 year. There was no significant difference in accelerometry-determined physical activity between the three trial arms at 1 year [22]. Consequently, a cohort analysis was conducted after pooling data from the three trial arms. Participants with valid accelerometry data at 1 and 7 years of follow-up (referred to below as baseline and follow-up, respectively) ( $n=202)$ constituted the sampling frame for this study, as this provided the optimal combination in terms of follow-up time and number of participants with complete accelerometry data. A final sample of 171 participants was included in analyses, after excluding those with missing cardiometabolic variables $(n=23)$, covariates $(n=6)$ or unrealistic data (television viewing time $>$ objectively measured sedentary time; $n=1$ ). We also excluded 1 person who became a wheelchair user during follow-up. All participants provided written informed consent and ethical approval was obtained from the Cambridge Central Research Ethics Committee (reference number: 09/110308/3). All investigations were carried out in accordance with the principles of the Declaration of Helsinki.

Outcome: cardiometabolic risk Data-collection procedures were the same at baseline and follow-up [21, 23]. In brief, participants attended the study centre, following an overnight fast, where a venous blood sample was collected. Standardised procedures were used to determine fasting plasma glucose (hexokinase method), triacylglycerol and HDL-cholesterol (standard enzymatic methods) and serum insulin levels (1235 AutoDELFIA immunoassay system, DAKO, Ely, UK) [23]. Trained personnel measured weight on standard calibrated scales and height using a rigid stadiometer with participants wearing light clothing. Waist circumference was measured twice at the mid-point between the lower costal margin and the level of the anterior superior iliac crests. Systolic and diastolic blood pressures were measured in seated participants three times at 1 min intervals using an automated Accutorr sphygmomanometer (Accutorr, Cambridge, UK). The average of multiple assessments was used. 
A clustered cardiometabolic risk score (CCMR) was computed [24, 25], incorporating indicators of central obesity (waist circumference), dyslipidaemia (triacylglycerol and HDL-cholesterol), hypertension (systolic and diastolic blood pressure) and hyperglycaemia (fasting plasma glucose and serum insulin). Individual variables were standardised (i.e. $z$ scores were computed: $z=[$ value - mean $] / S D)$, after normalising $\left(\log _{10}\right)$ triacylglycerol, glucose and insulin. Sexspecific baseline means and SDs from all participants with complete data for each cardiometabolic variable at baseline were used to standardise the baseline and follow-up variables. Subsequently, individual $z$ scores were summed, after inverting HDL-cholesterol $z$ scores and averaging systolic and diastolic blood pressure $z$ scores, and divided by the number of variables included. The CCMR was also calculated without the adiposity component $\left(\mathrm{CCMR}_{n o}\right.$ adip $)$, which allowed us to examine a potential mediation effect by change in adiposity for the associations with clustered cardiometabolic risk.

Exposure: objectively measured time spent sedentary and in MVPA, and self-reported television viewing time Physical activity was measured using the ActiGraph accelerometer (Manufacturing Technology, Fort Walton Beach, FL, USA), worn for 4 consecutive days at baseline and follow-up, as previously described [23]. ActiGraph models 7164 and GT1M were used at baseline and follow-up, respectively, providing a relatively comparable intensity classification of free-living activities [26, 27]. Continuous strings of zeros lasting for longer than $60 \mathrm{~min}$ were classified as non-wear time. Participants with fewer than three valid wear days (each including $>500 \mathrm{~min}$ of valid wear time) at baseline or followup were excluded $(n=21)$. Sedentary time was defined using a cut-off of $<100$ counts/min [28], and time spent in MVPA using a cut-off of $\geq 1,952$ counts/min [29]. A customised program (MAHUffe; www.mrc-epid.cam.ac.uk) was used for data cleaning and reduction. Time spent watching television and video (h/day) was self-reported using the EPAQ2 questionnaire, which has high repeatability and sufficient validity for ranking individuals [30].

Confounding variables Participants completed another standardised questionnaire, which asked about smoking (current/former/never), age at finishing full-time education as a marker of socioeconomic status (SES; younger/older than 16 years) and medication use for dyslipidaemia, hypertension or dysglycaemia (yes/no).

Statistical analysis All analyses were conducted using IBM SPSS Statistics 19 (SPSS, Chicago, IL, USA) and Stata 12.0 (Stata, College Station, TX, USA), and statistical significance was set at $p<0.05$. Descriptive characteristics (means $\pm \mathrm{SD}$ ) were compared between baseline and follow-up (paired $t$ tests), and between sexes and those who were included and excluded in the current study (independent $t$ tests). Changes in exposure and outcome variables were calculated as follow-up minus baseline. Change in fasting plasma glucose was normalised $\left(\log _{10}\right)$.

Multiple linear regression was used to examine the association between change in the exposure variables (objectively measured time spent sedentary and in MVPA, and television viewing time) with change in cardiometabolic risk over 6 years. The results are presented as unstandardised and standardised regression coefficients $(95 \% \mathrm{CI})$, following standardisation of both exposure and outcome variables. Unstandardised regression coefficients enable inference of the effect size in the original units of the exposure and outcome variables, whereas standardised regression coefficients enable direct comparison of the effect sizes across the exposure and outcome variables, as they are all expressed in SD units. Models were initially (Model A) adjusted for age, sex, SES, baseline exposure and the cardiometabolic risk variables under study; baseline and change in smoking status; change in monitor wear time and in dyslipidaemia, hypertension or dysglycaemia medication (as applicable); and follow-up time. Subsequently, we added baseline and change in objective MVPA (when change in sedentary time or television viewing was the exposure) or in sedentary time (when change in MVPA was the exposure) to these models (Model B). The variance inflation factor did not exceed 5 in any of the models, indicating absence of multicollinearity. No significant interactions between any of the exposures and sex were found, so the results are shown for both sexes combined. Including intervention arm in the analyses resulted in very similar associations in terms of their magnitude and direction. This covariate was therefore not included in the final models.

The mediating role of change in central and general adiposity was examined by means of the product of coefficients $\left(a^{*} b\right)$ method by MacKinnon et al [31], based on the regression coefficient between the exposure and mediator $(a)$ and between the mediator and outcome $(b)$. ' $a$ '-path coefficients were adjusted for all covariates in Model B. ' $b$ '-path coefficients were adjusted for all covariates in Model B, including baseline and change in the relevant exposure. Bootstrap analysis based on $>1,000$ replications was used to derive $95 \%$ CIs for the mediated effect [31].

\section{Results}

Descriptive characteristics Participants (45.6\% men, 41.5\% left school aged $\leq 16$ years) were followed for $6.27 \pm 0.46$ years (mean $\pm \mathrm{SD}$ ). At baseline, $40.4 \%$ of participants met the current MVPA guidelines ( $\geq 30 \mathrm{~min} /$ day), $47.4 \%$ were sedentary for $\geq 8 \mathrm{~h} /$ day, $57.3 \%$ watched television for $\geq 2 \mathrm{~h} /$ day, $24.0 \%$ were 
normal weight, $47.4 \%$ were overweight and $28.6 \%$ were obese. As shown in Table 1, mean values for CCMR, $\mathrm{CCMR}_{\text {no adip }}$, systolic blood pressure, fasting plasma glucose, fasting serum insulin, sedentary time and television viewing time increased from baseline to 6 years of follow-up. The percentage of wear time spent sedentary increased $(58.0 \pm 9.5 \%$ vs $63.0 \pm 9.0 \% ; p<$ 0.001 ) and percentage of wear time spent in MVPA decreased $(3.7 \pm 2.3 \%$ vs $3.3 \pm 2.5 \% ; p<0.05)$ from baseline to follow-up. Overall, $79 \%$ of participants increased their sedentary time and $44 \%$ increased their MVPA; $8 \%$ changed their smoking behaviour. Compared with women, men had an adverse risk profile for the majority of cardiometabolic risk indicators at baseline and follow-up (Electronic Supplementary Material [ESM] Table 1). Women reported watching more television and engaged in less MVPA but showed lower levels of objectively measured sedentary time compared with men; these sex differences were only statistically significant for sedentary and MVPA time at 6 years of follow-up. Compared with those who were excluded, participants included in the current study were slightly more educated $(p<0.05)$, but were similar in terms of age, BMI, individual cardiometabolic risk factors, sedentary time, MVPA and television viewing time at baseline.

Television viewing constituted $30.7 \pm 14.5 \%$ and $29.8 \pm$ $14.2 \%$ of objectively measured sedentary time at baseline and follow-up, respectively. Television viewing, sedentary and MVPA time showed relatively weak correlations at both time points (Spearman's rho: sedentary-MVPA: baseline: -0.18 ; follow-up: -0.19 ; sedentary-television: baseline: 0.15; follow-up: 0.05; MVPA-TV: baseline: -0.12 ; followup: -0.26). Correlations between change in exposure variables from baseline to follow-up were also relatively weak (sedentary-MVPA: -0.15 ; sedentary-TV: -0.05 ; MVPA-TV: -0.13 ). All three variables showed moderate tracking, as indicated by correlations between their baseline and followup levels (sedentary: 0.49; MVPA: 0.51; television: 0.68) [32].

Multiple linear regression analysis Unstandardised and standardised regression coefficients are shown in Table 2 and ESM Table 2, respectively. Greater increase in sedentary time was independently associated with a larger increase in $\mathrm{CCMR}, \mathrm{CCMR}_{\text {no adip }}$, waist circumference and triacylglycerol after adjusting for relevant confounders in Model A, albeit not quite reaching statistical significance for triacylglycerol $(p=$ 0.051). Adjustment for baseline and change in MVPA (Model B) attenuated the association for waist circumference, but not for CCMR, $\mathrm{CCMR}_{\text {no adip }}$ and triacylglycerol. Change in waist circumference and change in BMI did not mediate the associations with $\mathrm{CCMR}_{n o}$ adip and triacylglycerol $(a * b$ [95\% CI]: waist circumference: $\mathrm{CCMR}_{\text {no adip }}: 0.03[-0.01$, 0.07], triacylglycerol: 0.01 [-0.01, 0.04]; BMI: $\mathrm{CCMR}_{\text {no adip }}$ : 0.01 [-0.01, 0.02], triacylglycerol: $0.03[-0.02,0.08]$ ).

Greater decrease in MVPA time was associated with a larger increase in CCMR, $\mathrm{CCMR}_{n o}$ adip, waist circumference and fasting serum insulin, independent of relevant confounders in Model A. All of these associations, except for waist circumference, were attenuated after adjustment for baseline and change in sedentary time (Model B).

Although a greater increase in television viewing time was associated with a greater increase in CCMR, waist circumference, fasting plasma glucose and fasting serum insulin in Model A, these associations were all attenuated after adjustment for objectively measured baseline and change in MVPA (Model B).

Figure 1 displays the estimated marginal means (SE) for change in CCMR in six groups of participants defined by their change in sedentary time (decreased sedentary time [21\%],
Table 1 Descriptive characteristics of participants at baseline and after 6 years of follow-up

Data are means $\pm \mathrm{SD}$, unless otherwise indicated

${ }^{\text {a }}$ Geometric mean $\pm \mathrm{SD}$

${ }^{*} p<0.05 ;{ }^{* *} p<0.01$;

$* * * p<0.001$ between baseline and follow-up

\begin{tabular}{llll}
\hline Characteristic & Baseline & 6 year follow-up & Change \\
\hline Age (years) & $42.5 \pm 6.3$ & $48.8 \pm 6.3^{* * *}$ & $6.3 \pm 0.5$ \\
Body mass index $\left(\mathrm{kg} / \mathrm{m}^{2}\right)$ & $28.0 \pm 4.8$ & $28.0 \pm 4.8$ & $0.0 \pm 0.2$ \\
CCMR & $0.0 \pm 0.7$ & $0.1 \pm 0.8^{*}$ & $0.1 \pm 0.5$ \\
CCMR $_{\text {no adip }}$ & $0.0 \pm 0.7$ & $0.1 \pm 0.8^{*}$ & $0.1 \pm 0.5$ \\
Waist circumference (cm) & $94.2 \pm 12.6$ & $94.9 \pm 13.5$ & $0.7 \pm 7.2$ \\
Triacylglycerol (mmol/) & $1.3 \pm 0.4^{\mathrm{a}}$ & $1.2 \pm 0.4^{\mathrm{a}}$ & $-0.1 \pm 1.1$ \\
HDL-cholesterol (mmol/l) & $1.4 \pm 0.4$ & $1.4 \pm 0.4$ & $0.0 \pm 0.3$ \\
Systolic blood pressure (mmHg) & $119.4 \pm 12.6$ & $122.7 \pm 13.3^{* *}$ & $3.3 \pm 13.0$ \\
Diastolic blood pressure (mmHg) & $75.8 \pm 9.7$ & $76.3 \pm 9.4$ & $0.5 \pm 9.2$ \\
Fasting plasma glucose (mmol/l) & $5.0 \pm 0.1^{\mathrm{a}}$ & $5.3 \pm 0.2^{\mathrm{a} * * *}$ & $0.4 \pm 1.4$ \\
Fasting serum insulin (pmol/l) & $54.4 \pm 0.8^{\mathrm{a}}$ & $49.5 \pm 1.0^{\mathrm{a} *}$ & $-1.0 \pm 41.5$ \\
Sedentary time (h/day) & $7.9 \pm 1.5$ & $9.1 \pm 1.7^{* * *}$ & $1.2 \pm 1.7$ \\
MVPA (h/day) & $0.5 \pm 0.3$ & $0.5 \pm 0.4$ & $0.0 \pm 0.4$ \\
Television viewing time (h/day) & $2.4 \pm 1.1$ & $2.6 \pm 1.2^{* * *}$ & $0.3 \pm 0.9$ \\
Monitor wear time (h/day) & $13.6 \pm 1.3$ & $14.5 \pm 1.4^{* * *}$ & $0.9 \pm 1.7$ \\
\hline
\end{tabular}


Table 2 Associations between change in sedentary time, MVPA and television viewing time (all h/day) over 6 years and changes in cardiometabolic risk from baseline to 6 year follow-up

\begin{tabular}{|c|c|c|c|c|}
\hline Cardiometabolic outcome & Model & Sedentary time & MVPA time & Television viewing time \\
\hline \multirow[t]{2}{*}{$\mathrm{CCMR}^{\mathrm{a}, \mathrm{b}, \mathrm{c}}$} & $\mathrm{A}$ & $0.11(0.04,0.17)^{* *}$ & $-0.33(-0.57,-0.09)^{* *}$ & $0.09(0.01,0.19)^{*}$ \\
\hline & $\mathrm{B}$ & $0.08(0.01,0.15)^{*}$ & $-0.20(-0.46,0.05)$ & $0.08(-0.01,0.18)$ \\
\hline \multirow[t]{2}{*}{$\mathrm{CCMR}_{\text {no adip }}^{\mathrm{a}, \mathrm{b}, \mathrm{c}}$} & A & $0.10(0.03,0.17)^{* *}$ & $-0.31(-0.57,-0.06)^{*}$ & $0.09(-0.01,0.19)$ \\
\hline & $\mathrm{B}$ & $0.08(0.01,0.16)^{*}$ & $-0.19(-0.46,0.09)$ & $0.08(-0.03,0.18)$ \\
\hline \multirow[t]{2}{*}{ Waist circumference $(\mathrm{cm})$} & A & $1.39(0.44,2.33)^{* *}$ & $-5.17(-8.60,-1.74)^{* *}$ & $1.49(0.13,2.86)^{*}$ \\
\hline & $\mathrm{B}$ & $0.93(-0.08,1.95)$ & $-3.86(-7.58,-0.14)^{*}$ & $1.24(-0.14,2.63)$ \\
\hline \multirow[t]{2}{*}{ Triacylglycerol $^{\mathrm{a}}(\mathrm{mmol} / \mathrm{l})$} & A & $0.13(-0.01,0.26)$ & $-0.01(-0.48,0.48)$ & $0.11(-0.08,0.29)$ \\
\hline & $\mathrm{B}$ & $0.15(0.01,0.29)^{*}$ & $0.21(-0.31,0.72)$ & $0.10(-0.09,0.30)$ \\
\hline \multirow[t]{2}{*}{ HDL-cholesterol $^{\mathrm{a}}(\mathrm{mmol} / \mathrm{l})$} & A & $-0.01(-0.04,0.02)$ & $0.09(-0.02,0.21)$ & $-0.01(-0.06,0.03)$ \\
\hline & $\mathrm{B}$ & $-0.01(-0.04,0.03)$ & $0.09(-0.04,0.21)$ & $-0.01(-0.06,0.04)$ \\
\hline \multirow[t]{2}{*}{ Systolic blood pressure ${ }^{\mathrm{b}}(\mathrm{mmHg})$} & $\mathrm{A}$ & $0.52(-1.04,2.07)$ & $0.81(-4.96,6.58)$ & $-0.07(-2.31,2.17)$ \\
\hline & $\mathrm{B}$ & $0.73(-0.97,2.43)$ & $2.16(-4.11,8.43)$ & $-0.14(-2.48,2.21)$ \\
\hline \multirow[t]{2}{*}{ Diastolic blood pressure ${ }^{\mathrm{b}}(\mathrm{mmHg})$} & A & $0.70(-0.37,1.77)$ & $-1.63(-5.57,2.31)$ & $-0.08(-1.63,1.48)$ \\
\hline & $\mathrm{B}$ & $0.61(-0.56,1.77)$ & $-0.69(-4.98,3.61)$ & $-0.20(-1.81,1.42)$ \\
\hline \multirow[t]{2}{*}{ Fasting plasma glucose $\mathrm{e}^{\mathrm{c}}(\mathrm{mmol} / \mathrm{l})$} & A & $0.01(-0.01,0.01)$ & $-0.02(-0.06,0.02)$ & $0.02(0.01,0.03)^{*}$ \\
\hline & $\mathrm{B}$ & $0.01(-0.01,0.01)$ & $-0.02(-0.06,0.02)$ & $0.01(-0.01,0.03)$ \\
\hline \multirow[t]{2}{*}{ Fasting serum insulin ${ }^{\mathrm{c}}(\mathrm{pmol} / \mathrm{l})$} & $\mathrm{A}$ & $4.80(-0.53,10.12)$ & $-21.57(-40.92,-2.23)^{*}$ & $7.68(0.02,15.34)^{*}$ \\
\hline & $\mathrm{B}$ & $2.85(-2.91,8.62)$ & $-17.25(-38.34,3.84)$ & $7.24(-0.63,15.10)$ \\
\hline
\end{tabular}

Data are unstandardised regression coefficients $(95 \% \mathrm{CI})$

Model A: adjusted for baseline age, sex, SES, exposure under study and cardiometabolic risk variable under study; baseline and change in smoking status; change in monitor wear time and in medication for ${ }^{\mathrm{a}}$ dyslipidaemia, ${ }^{\mathrm{b}}$ hypertension or ${ }^{\mathrm{c}}$ dysglycaemia; and follow-up time

Model B: adjusted for all covariates in Model A plus baseline and change in MVPA/sedentary time

$* p<0.05 ; * *<<0.01$

increased sedentary time by $<2 \mathrm{~h} /$ day [54\%], increased sedentary time by $\geq 2 \mathrm{~h} /$ day [25\%]) and their change in MVPA

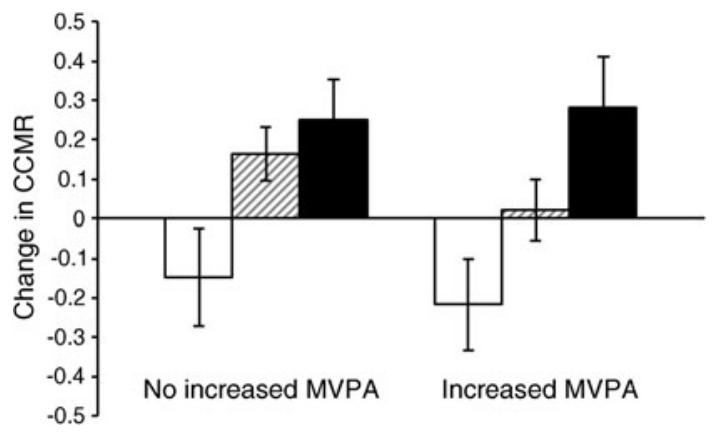

Fig. 1 Change in clustered cardiometabolic risk according to change in sedentary time (three groups) and change in MVPA time (two groups). Data are estimated marginal means (SE), adjusted for baseline time spent sedentary, baseline MVPA time, baseline clustered cardiometabolic risk, age, sex and SES; baseline and change in smoking status; change in monitor wear time and in medication for dyslipidaemia, hypertension and dysglycaemia; and follow-up time. White bars represent participants who decreased their sedentary time; grey striped bars represent those who increased their sedentary time by $<2 \mathrm{~h} /$ day; and black bars represent those who increased their sedentary time by $\geq 2 \mathrm{~h}$ /day. The three left bars represent participants who did not increase their MVPA time, while the three right bars represent participants who increased their MVPA time. Change is follow-up minus baseline (did not increase MVPA [56\%], increased MVPA [44\%]). There was no interaction between change in sedentary and MVPA times and change in CCMR. Assuming causality, this suggests that the effect of changing sedentary time on CCMR would be similar among participants who did and did not increase their MVPA during follow-up. In addition, no interaction was found between change in sedentary and MVPA times and change in $\mathrm{CCMR}_{\text {no adip }}$ or triacylglycerol.

\section{Discussion}

Increasing sedentary time was independently associated with increasing clustered cardiometabolic risk and triacylglycerol over 6 years in adults with a parental history of type 2 diabetes. Increased MVPA was associated with reduced waist circumference. As one of the first longitudinal studies to examine the associations between sedentary time and cardiometabolic risk in adults at high diabetes risk, we have highlighted the potential benefits of targeting a reduction in sedentary time in future interventions. In context, based on our findings for triacylglycerol levels, decreasing sedentary time by $2 \mathrm{~h} /$ day would be associated with an approximately $7 \%$ 
lower risk of cardiovascular events [33]. This is a feasible reduction in sedentary time, as indicated by a recent lifestyle intervention [34].

Our findings extend previous cross-sectional findings in other adult populations of the independent associations between sedentary time and clustered cardiometabolic risk [35] and triacylglycerol levels [6]. The association with change in HDL-cholesterol did not reach statistical significance in our study, although it was in the hypothesised direction, which is consistent with cross-sectional findings from the Health Survey for England [7]. It is, however, in disagreement with the National Health and Nutrition Examination Survey, in which a significant cross-sectional association was found with HDL-cholesterol (albeit most strongly in men and non-Hispanic whites) [6], as well as with the findings from Cooper et al showing a prospective association with HDL-cholesterol in English adults with newly diagnosed type 2 diabetes [14]. Evidence that sedentary time might influence waist circumference and glucose metabolism is also mixed, in both cross-sectional $[6,7,23$, 35-37] and longitudinal studies [14, 17, 38-40]. Although physiological mechanisms need further clarification [19], some of the inconsistent findings to date may, in part, be explained by differences in the populations studied (e.g. health risk status, ethnicity [6] and age) as well as differences in the underlying confounding structures. Our non-significant findings for blood pressure are in agreement with the results from most previous studies [6, 7, 23, 35].

Few significant independent associations were found for change in MVPA in our study. This may indicate that solely focusing on increasing MVPA, a potentially challenging lifestyle target for high-risk individuals [4], may not be the most effective strategy for future interventions [23]. The lack of associations may, however, be explained to some extent by the limited change in MVPA between baseline and follow-up. The relatively small sample size may also partly account for the lack of significant findings, not only for MVPA, but also for the other exposure variables. Nevertheless, even though our findings were not statistically significant, they were generally in the hypothesised directions. In addition, the strengths of the associations are consistent with those from previous studies. Healy et al, for example, showed that sedentary time was more strongly associated with triacylglycerol levels than with HDL-cholesterol levels, and with serum insulin as compared with fasting plasma glucose [6]. Change in central obesity is a potential mediator [20], but we found no evidence that these associations were mediated by changes in general and central adiposity. An alternative biologically plausible mechanism underpinning the detrimental effects of prolonged sitting on triacylglycerol involves reduced lipoprotein lipase activity as a result of fewer skeletal muscle contractions [41].
Individuals at high risk of diabetes enrolled in lifestyle intervention programmes demonstrate limited success in increasing their MVPA levels [4]. Replacing sedentary time with light-intensity activities that are part of daily living might be an important intervention target. The vast majority of the waking day is spent either sedentary or in light-intensity activity [35], and changes in time spent sedentary and in light-intensity activity over 6 years were highly correlated $(-0.47)$. This population thus makes most of its transfers in terms of time use between activities of light and sedentary intensity. The importance of total daily physical activity, rather than MVPA alone, on cardiometabolic risk has been shown before, specifically in high-risk adults [23].

The significant associations between change in television viewing and clustered cardiometabolic risk, waist circumference, fasting plasma glucose and serum insulin were attenuated after adjustment for objectively measured MVPA, indicating they are not independent of MVPA. This may suggest that previous associations between television viewing time and cardiometabolic health, which adjusted for MVPA measured by self-report and were sometimes restricted to leisure-time MVPA only [5, 8, 15, 16, 18, 42], may have been affected by residual confounding for this key variable. Future investigations of the health implications of sedentary behaviour are therefore encouraged to use objective measures of physical activity.

The differential associations for television viewing and sedentary time with cardiometabolic risk found in the current study indicate that findings for television viewing should not be generalised to overall sedentary time. Television viewing in this population represented less than a third of objectively measured sedentary time and correlations between both indicators were weak. In contrast, two previous investigations showed stronger associations between self-reported television viewing time and cardiometabolic risk compared with objectively measured sedentary time, albeit derived from cross-sectional data $[7,36]$. A study in adults aged $\geq 60$ years [36], who on average watched more television than younger age groups, showed stronger associations between television viewing and objectively measured sedentary time compared with younger age groups [43]. Future large longitudinal cohort studies covering a wide age range, and also including a whole set of cardiometabolic risk indicators and appropriately accounting for confounding factors, are needed to further explore the differential role of television viewing and sedentary time in determining cardiometabolic health.

To the best of our knowledge, this is the first longitudinal study simultaneously examining the associations of total sedentary time, television viewing time and MVPA time with clustered cardiometabolic risk and a wide set of individual risk factors. All risk factors were used continuously, which optimised statistical power. It is unclear whether participants changed their sedentary time before any changes in 
cardiometabolic risk, which hampers inferences on the direction of causality. Change in waist circumference has been shown to predict an increase in sedentary time in a healthy, middle-aged white population [40]. However, unlike most previous research, our change models examined withinparticipant effects, which are less prone to unmeasured timeindependent confounders (e.g. genetic factors) because each individual acts as his/her own control. Other major strengths of this study include the objective measurement of two of the three exposures, which substantially reduced measurement error. Participants were followed for longer than 6 years. Finally, the mediation effect of general and central adiposity was also explored using the product of coefficient method [31].

The following limitations should, however, be considered. Participants included in the current study were slightly more educated than those who were excluded, but did not differ in terms of cardiometabolic, sedentary or activity profile, BMI, sex or age. Nevertheless, the relatively small sample of white adults with a high risk for type 2 diabetes included in this study might limit the external validity of the observed associations. Future longitudinal studies in more heterogeneous populations should explore the relative importance of a change in these three behaviours for cardiometabolic health. Television viewing time was self-reported, which confers greater measurement error and is likely to have attenuated any associations, compared with objectively measured exposures. However, assuming participants' misreporting behaviour would remain constant between baseline and follow-up, a change in television viewing should be captured with lower measurement error compared with television viewing time measured at just one time point.

Participants wore the accelerometer for 4 consecutive days, not necessarily including a weekend day. Sedentary and MVPA time might therefore not be completely reflective of participants' habitual long-term behaviour. Despite controlling for several potential confounding variables, some findings could still be partially explained by other factors that were not measured, such as alcohol intake, employment status and type of work, or only partially measured (e.g. SES by means of age at finishing full-time education). Given the lack of dietary information, the mediating role of snacking and overall dietary quantity and quality, which are especially relevant for television viewing, could not be explored. Finally, to examine our research question, we conducted a number of significance tests, which might have increased the risk of type 1 errors. Future longitudinal research will help to confirm our findings.

In conclusion, in adults at high risk for type 2 diabetes, increasing sedentary time was independently associated with increasing clustered cardiometabolic risk and triacylglycerol levels. Replacing sedentary time by light-intensity activity, and thus increasing overall daily physical activity levels, could be an important behavioural target in these high-risk individuals. Further longitudinal observational studies are now needed to extend these findings to other populations that are heterogeneous in terms of age, ethnicity and health status, as well as intervention studies, both physiological and freeliving, examining acute and longer-term effects to further investigate the direction and mechanism of causality.

Acknowledgements The ProActive research team includes, besides the authors, K. M. Williams (study coordination, Primary Care Unit, University of Cambridge, UK), J. Grant (study support, Primary Care Unit, Cambridge, UK), A. T. Prevost (study statistician, Primary Care Unit), W. Hollingworth (study economist, University of Bristol, Bristol, UK), N. J. Wareham (principal investigator, MRC Epidemiology Unit, Cambridge, UK), D. Spiegelhalter (principal investigator, MRC Biostatistics Unit, Cambridge, UK), W. Hardeman (principal investigator, Primary Care Unit), S. Sutton (principal investigator, Primary Care Unit), and A. L. Kinmonth (principal investigator, Primary Care Unit). We thank the study participants and practice teams for their collaboration and work in helping with recruitment.

Funding This work was supported by the UK Medical Research Council (grant no. ISRCTN 61323766); UK National Health Service Research and Development; the UK Royal College of General Practitioners Scientific Foundation; Diabetes UK (grant no. RG35259); the British Heart Foundation (grant no. FS/12/58/29709 to KW); and the National Institute for Health Research School for Primary Care Research (to SJG). The views expressed in this publication are those of the authors and not necessarily those of the National Health Service, the National Institute for Health Research or the UK Department of Health.

Access to research materials Data are not currently available for public release.

Duality of interest The authors declare that there is no duality of interest associated with this manuscript.

Author contributions KW wrote the manuscript and is the guarantor. UE, RKS, GO and SJG (principal investigator) acquired and coded the data. KW and SJS analysed the data. KW, UE, SB and RKS interpreted the data. All authors revised the article for important intellectual content and approved the final version of the manuscript.

Open Access This article is distributed under the terms of the Creative Commons Attribution License which permits any use, distribution, and reproduction in any medium, provided the original author(s) and the source are credited.

\section{References}

1. Mottillo S, Filion KB, Genest J et al (2010) The metabolic syndrome and cardiovascular risk a systematic review and meta-analysis. J Am Coll Cardiol 56:1113-1132

2. Ford ES, Li C, Sattar N (2008) Metabolic syndrome and incident diabetes: current state of the evidence. Diabetes Care 31:1898-1904

3. Haskell WL, Lee IM, Pate RR et al (2007) Physical activity and public health: updated recommendation for adults from the American College of Sports Medicine and the American Heart Association. Med Sci Sports Exerc 39:1423-1434

4. Yates T, Khunti K, Bull F, Gorely T, Davies MJ (2007) The role of physical activity in the management of impaired glucose tolerance: a systematic review. Diabetologia 50:1116-1126 
5. Wijndaele K, Duvigneaud N, Matton L et al (2009) Sedentary behaviour, physical activity and a continuous metabolic syndrome risk score in adults. Eur J Clin Nutr 63:421-429

6. Healy GN, Matthews CE, Dunstan DW, Winkler EA, Owen N (2011) Sedentary time and cardio-metabolic biomarkers in US adults: NHANES 2003-06. Eur Heart J 32:590-597

7. Stamatakis E, Hamer M, Tilling K, Lawlor DA (2012) Sedentary time in relation to cardio-metabolic risk factors: differential associations for self-report vs accelerometry in working age adults. Int J Epidemiol 41:1328-1337

8. Pinto Pereira SM, Ki M, Power C (2012) Sedentary behaviour and biomarkers for cardiovascular disease and diabetes in mid-life: the role of television-viewing and sitting at work. PLoS One 7:e31132

9. Celis-Morales CA, Perez-Bravo F, Ibanez L, Salas C, Bailey ME, Gill JM (2012) Objective vs. self-reported physical activity and sedentary time: effects of measurement method on relationships with risk biomarkers. PLoS One 7:e36345

10. Sedentary Behaviour Research Network (2012) Standardized use of the terms "sedentary" and "sedentary behaviours". Appl Physiol Nutr Metab 37:540-542

11. Matthews CE, Chen KY, Freedson PS et al (2008) Amount of time spent in sedentary behaviors in the United States, 2003-2004. Am J Epidemiol 167:875-881

12. Craft LL, Zderic TW, Gapstur SM et al (2012) Evidence that women meeting physical activity guidelines do not sit less: an observational inclinometry study. Int J Behav Nutr Phys Act 9:122

13. Thorp AA, Owen N, Neuhaus M, Dunstan DW (2011) Sedentary behaviors and subsequent health outcomes in adults: a systematic review of longitudinal studies, 1996-2011. Am J Prev Med 41:207215

14. Cooper AR, Sebire S, Montgomery AA et al (2012) Sedentary time, breaks in sedentary time and metabolic variables in people with newly diagnosed type 2 diabetes. Diabetologia 55:589-599

15. Wijndaele K, Healy GN, Dunstan DW et al (2010) Increased cardiometabolic risk is associated with increased TV viewing time. Med Sci Sports Exerc 42:1511-1518

16. Stamatakis E, Hamer M, Mishra GD (2012) Early adulthood television viewing and cardiometabolic risk profiles in early middle age: results from a population, prospective cohort study. Diabetologia 55:311-320

17. Lahjibi E, Heude B, Dekker JM et al (2013) Impact of objectively measured sedentary behaviour on changes in insulin resistance and secretion over 3 years in the RISC study: interaction with weight gain. Diabetes Metab 39:217-225

18. Thorp AA, Healy GN, Owen N et al (2010) Deleterious associations of sitting time and television viewing time with cardiometabolic risk biomarkers: Australian Diabetes, Obesity and Lifestyle (AusDiab) study 2004-2005. Diabetes Care 33:327-334

19. Tremblay MS, Colley RC, Saunders TJ, Healy GN, Owen N (2010) Physiological and health implications of a sedentary lifestyle. Appl Physiol Nutr Metab 35:725-740

20. Alberti KGM, Zimmet P, Shaw J (2005) The metabolic syndrome-a new worldwide definition. Lancet 366:1059-1062

21. Williams K, Prevost AT, Griffin S et al (2004) The ProActive trial protocol - a randomised controlled trial of the efficacy of a family-based, domiciliary intervention programme to increase physical activity among individuals at high risk of diabetes [ISRCTN61323766]. BMC Public Health 4:48

22. Kinmonth AL, Wareham NJ, Hardeman W et al (2008) Efficacy of a theory-based behavioural intervention to increase physical activity in an at-risk group in primary care (ProActive UK): a randomised trial. Lancet 371:41-48

23. Ekelund U, Griffin SJ, Wareham NJ (2007) Physical activity and metabolic risk in individuals with a family history of type 2 diabetes. Diabetes Care 30:337-342
24. Wijndaele $\mathrm{K}$, Brage $\mathrm{S}$, Besson $\mathrm{H}$ et al (2011) Television viewing time and incident cardiovascular disease: prospective associations and mediation analysis in the EPIC Norfolk Study. PLoS One 6:e20058

25. Brage S, Wedderkopp N, Ekelund U et al (2004) Features of the metabolic syndrome are associated with objectively measured physical activity and fitness in Danish children - the European Youth Heart Study (EYHS). Diabetes Care 27:2141-2148

26. Kozey SL, Staudenmayer JW, Troiano RP, Freedson PS (2010) Comparison of the ActiGraph 7164 and the ActiGraph GT1M during self-paced locomotion. Med Sci Sports Exerc 42:971-976

27. John D, Tyo B, Bassett DR (2010) Comparison of four ActiGraph accelerometers during walking and running. Med Sci Sports Exerc 42:368-374

28. Healy GN, Clark BK, Winkler EA, Gardiner PA, Brown WJ, Matthews CE (2011) Measurement of adults' sedentary time in population-based studies. Am J Prev Med 41:216-227

29. Freedson PS, Melanson E, Sirard J (1998) Calibration of the Computer Science and Applications, Inc. accelerometer. Med Sci Sports Exerc 30:777-781

30. Wareham NJ, Jakes RW, Rennie KL, Mitchell J, Hennings S, Day NE (2002) Validity and repeatability of the EPIC-Norfolk Physical Activity Questionnaire. Int J Epidemiol 31:168-174

31. MacKinnon DP, Fairchild AJ, Fritz MS (2007) Mediation analysis. Annu Rev Psychol 58:593-614

32. Malina RM (2001) Tracking of physical activity across the lifespan. Res Digest 3:3-6

33. Hokanson JE, Austin MA (1996) Plasma triglyceride level is a risk factor for cardiovascular disease independent of high-density lipoprotein cholesterol level: a meta-analysis of population-based prospective studies. J Cardiovasc Risk 3:213-219

34. Healy GN, Eakin EG, Lamontagne AD et al (2013) Reducing sitting time in office workers: short-term efficacy of a multicomponent intervention. Prev Med 57:43-48

35. Healy GN, Wijndaele K, Dunstan DW et al (2008) Objectively measured sedentary time, physical activity, and metabolic risk: the Australian Diabetes, Obesity and Lifestyle Study (AusDiab). Diabetes Care 31:369-371

36. Stamatakis E, Davis M, Stathi A, Hamer M (2012) Associations between multiple indicators of objectively-measured and selfreported sedentary behaviour and cardiometabolic risk in older adults. Prev Med 54:82-87

37. Healy GN, Dunstan DW, Salmon J et al (2007) Objectively measured light-intensity physical activity is independently associated with 2 -h plasma glucose. Diabetes Care 30:1384-1389

38. Helmerhorst HJF, Wijndaele K, Brage S, Wareham NJ, Ekelund U (2009) Objectively measured sedentary time predicts insulin resistance, independent of moderate and vigorous physical activity. Diabetes 58:1776-1779

39. Ekelund U, Brage S, Griffin SJ, Wareham NJ (2009) Objectively measured moderate- and vigorous-intensity physical activity but not sedentary time predicts insulin resistance in high-risk individuals. Diabetes Care 32:1081-1086

40. Ekelund U, Brage S, Besson H, Sharp S, Wareham NJ (2008) Time spent being sedentary and weight gain in healthy adults: reverse or bidirectional causality? Am J Clin Nutr 88:612-617

41. Hamilton MT, Hamilton DG, Zderic TW (2007) Role of low energy expenditure and sitting in obesity, metabolic syndrome, type 2 diabetes and cardiovascular disease. Diabetes 56:2655-2667

42. Hansen AL, Wijndaele K, Owen N et al (2012) Adverse associations of increases in television viewing time with 5year changes in glucose homoeostasis markers: the AusDiab study. Diabet Med 29:918-925

43. Clark BK, Healy GN, Winkler EAH et al (2011) Relationship of television time with accelerometer-derived sedentary time: NHANES. Med Sci Sports Exerc 43:822-828 